\title{
Sind 50-Stunden-Woche und zeitgerechte Weiterbildung miteinander vereinbar?
}

\author{
Gedanken eines Kinderchirurgen
}

\section{G. Schwöbel}

Am 1. Januar 2005 wird für alle Assistenzärztinnen und Assistenzärzte der Schweiz die 50-Stunden-Woche eingeführt. Sie beeinflusst nicht nur die Dienstpläne der Kliniken, sondern auch die Weiterbildungsordnungen (WBO) der Fachgesellschaften (FG). Während die FG der Grundversorger ihre WBO darauf stützen, wie lange ein Arzt oder eine Ärztin in einer Institution arbeitet, steht bei den interventionellen FG der Nachweis von selbst durchgeführten Eingriffen im Vordergrund.

Kleinere Kliniken, welche bisher ihre Notfallstationen nachts mit Pikettdiensten betreiben konnten, sind stärker betroffen als Kliniken mit einem grossen Assistentenpool. FG, welche ein breites Spektrum von verschiedenen Eingriffen zu vermitteln haben, können den vorgeschriebenen Operationskatalog nicht mehr zeitgerecht bieten. Am Beispiel der Kinderchirurgie wird gezeigt, dass die vorgesehene Arbeitszeitverkürzung nicht mehr aufgefangen werden kann und neue Weiterbildungsmodelle gesucht werden müssen.

Am 1. Januar 2005 wird in allen Schweizer Spitälern die 50-Stunden-Woche für Assistenzärztinnen- und Assistenzärzte eingeführt werden. Ausgenommen von dieser Regelung werden (vorläufig) noch die Kaderärzte sein, obwohl Bestrebungen bestehen, mindestens auch die Oberärzte unter das Reglement der 50-StundenWoche zu stellen. Zwei Hauptgründe haben zu dieser politischen Entscheidung geführt.

\section{Gründe für die 50-Stunden-Woche}

Zum einen der Sicherheitsaspekt, indem argumentiert wurde, dass müde Ärzte gefährliche Ärzte seien und Fehler dadurch vermieden werden könnten, dass nur ausgeruhte Ärzte in den Spitälern arbeiten. $\mathrm{Zu}$ diesem Argument muss gesagt werden, dass Assistenzärztinnen und Assistenzärzte in eine Hierarchie eingebunden sind und dass Oberärzte, Leitende Ärzte und Chefärzte dazu da sind, Fehler zu vermeiden helfen. Zudem müssten mit dem Argument der Gefährlichkeit gerade die obersten Kader nach 50 Stunden aus den Spitälern entfernt werden, da sie viel mehr Möglichkeiten haben, Patienten zu schaden als die Ärzte der untersten Hierarchiestufen.

Das zweite Argument kommt von den Assistenzärztinnen und Assistenzärzten selbst, welche andere Lebensentwürfe haben als die Generation vor ihnen und welche auch in der Weiterbildungsperiode nicht auf eine heute allgemein übliche Lebensqualität ausserhalb der Erwerbstätigkeit verzichten wollen. Da es sich bei den in Weiterbildung stehenden Ärztinnen und Ärzten aber um gut bezahlte Hochschulabsolventen handelt, welche primär für eine ärztliche Leistung und nicht für ihre Weiterbildung angestellt worden sind, muss neu definiert werden, wieviel der Zeit, welche sich die Ärzte im Spital aufhalten, unter Dienstleistung abgebucht werden kann und wieviel Zeit für die Weiterbildung aufgewendet wird.

\section{Weiterbildungsordnungen}

Die Weiterbildungsordnungen (WBO) der Fachgesellschaften sind in den letzten 10 Jahren einer grundsätzlichen Korrektur unterzogen worden. Weiterbildungsjahre wurden angepasst und Curricula neu erarbeitet. In den interventionellen Fächern wurde die Zahl und Art der Eingriffe neu definiert. Die Fachgesellschaften gingen davon aus, dass die bisher üblichen Arbeitszeiten Grundlage der Modelle sein sollten. Deshalb müssen die für die Weiterbildung verantwortlichen Vertreter der Fachgesellschaften sich die Frage stellen, ob auch unter der neuen Ordnung in der vorgegebenen Zeit die erwartete Weiterbildung möglich ist und ob die Qualität der Weiterbildung unter der Verkürzung der Arbeitszeit leidet.

Probleme mit der (WBO) unter veränderten Arbeitszeiten betreffen alle Fächer. Wir müssen uns aber darüber klar sein, dass einige Spezialarzttitel mehr und andere weniger betroffen sind. Besonders schwierig wird die Situation für die interventionellen Fächer, welche eine bestimmte Zahl von Eingriffen verschiedener Schweregrade und nicht nur eine Anzahl Weiterbildungsjahre vorweisen müssen. Unter ihnen sind die chirurgischen Fächer und damit auch die Kinderchirurgie besonders betroffen, welche 
bereits heute den Facharzttitel im Durchschnitt nicht, wie in der WBO verlangt, nach sechs Jahren, sondern nach frühestens neun Jahren verleiht.

Die Kinderchirurgie gehört zu der Gruppe von Fächern, welche sich sehr früh eine Weiterbildungsordnung gegeben hat und welche bereits seit 1988 jährlich eine Facharztprüfung durchführt. Wir können in bezug auf die Umsetzung unserer WBO also auf eine Erfahrung zurückblicken, welche anderen Fächern, darunter den grossen Fachgesellschaften der Grundversorger und der Psychiater, fehlt.

Die Kinderchirurgie ist die Spezialchirurgie am wachsenden Organismus. Da sie eng mit der allgemeinen Chirurgie verbunden ist, legen wir grossen Wert auf eine solide allgemeinchirurgische Grundausbildung und haben deshalb in unserer WBO festgelegt, dass der zukünftige Kinderchirurg mindestens zwei Jahre allgemeine Chirurgie zu absolvieren und das chirurgische Basisexamen zu bestehen hat. Da wir die $\mathrm{Zu}-$ sammenarbeit mit der Pädiatrie besonders fördern wollen und der Meinung sind, dass der Kinderchirurg ein pädiatrisches Grundwissen mitbringen muss, verlangen wir weiter sechs Monate Pädiatrie. Schliesslich kommen noch, wie für alle Chirurgen, drei Monate Anästhesie hinzu, so dass sich schliesslich die fachspezifische Weiterbildung auf $3 \frac{1}{4}$ Jahre beschränkt. Aus standespolitischen Gründen durfte ja in den letzten Jahren an der sechsjährigen Weiterbildungszeit nicht gerüttelt werden.

In 31/4 Jahren müssten also rund 500 mittlere bis grosse Eingriffe durchgeführt und das Fach Kinderchirurgie von Grund auf gelernt werden.

Der Leser kann sich denken, dass die Wirklichkeit anders aussieht. Viele junge Ärztinnen und Ärzte verlängern die unspezifische Weiterbildung, v.a. wenn sie auf der allgemeinen Chirurgie tätig sind, weswegen eine beträchtliche Zahl auch der jüngeren Kinderchirurgen den Facharzttitel für Chirurgie trägt. Zudem dauert die spezifische Weiterbildung im Durchschnitt deutlich länger als die verlangten 3/1/4 Jahre. Deswegen ist bisher kein Facharzttitel vor neun Jahren Weiterbildung vergeben worden. Und dies wohlgemerkt unter der alten Ordnung mit nach oben weitgehend unbeschränkter Arbeitszeit.

\section{Tätigkeit im Spital: Dienstleistung oder Weiterbildung?}

Welches sind die wichtigsten Tätigkeiten eines Assistenzarztes oder einer Assistenzärztin in einem öffentlichen Spital und in welchem zeit- lichen Zusammenhang stehen diese Tätigkeiten mit der Weiterbildungszeit?

Zum einen ist der Arzt in Weiterbildung Tages- und Nachtarzt in einer Notfallstation. Dabei handelt es sich einerseits um eine reine Pförtnerfunktion, da von einem öffentlichen Spital verlangt wird, dass Tag und Nacht unverzüglich ein Arzt zur Verfügung steht. Andererseits ist der Arzt der Notfallstation aber auch derjenige, welcher mit grösseren und kleineren Notfällen allein oder mit Hilfe des diensttuenden Oberarztes fertig werden muss.

Den meisten Notfallstationen ist ein Ambulatorium angegliedert, in welchem Patienten nachkontrolliert werden. In chirurgischen und internistischen Tageskliniken werden Patienten ambulant operiert oder über Stunden internistisch betreut. Schliesslich dienen Polikliniken dazu, Abklärungen vorzunehmen und Patienten zu beraten.

Der dritte Aufgabenkomplex ist die Arbeit auf der Station. Dazu gehören Betreuung von Eintritten und Austritten, Verordnungen von Medikamenten und Untersuchungen, Visiten, Gespräche und allgemeine Führungsaufgaben in enger Zusammenarbeit mit dem Pflegedienst. Spezifisch für Pädiatrie und Kinderchirurgie sind die Elterngespräche, welche oft zeitraubend sein können.

Während die oben beschriebenen Tätigkeiten für alle klinischen Fächer gelten, kommt für die chirurgischen Fächer die Arbeit im Operationssaal hinzu. Dabei handelt es sich um Assistenzen erster und zweiter Hand, Operationen unter Anleitung, selbständige Eingriffe und Lehreingriffe für jüngere Kolleginnen und Kollegen. Schliesslich wird, gerade in grösseren Spitälern, auch noch eine Mitarbeit bei wissenschaftlichen Projekten gewünscht.

Bis in die achtziger Jahre liefen die Tätigkeit in der Notfallstation und insbesondere der Nachtdienst und der Wochenenddienst nebenher und wurden höchstens pauschal finanziell abgegolten. Deshalb waren damals Arbeits- und Präsenzzeiten von 80 bis 90 Stunden pro Woche nichts Ungewöhnliches.

Die Einführung des Tag-/Nachtarzt-Systems in den neunziger Jahren brachte eine echte Erleichterung und eine deutliche Verbesserung der Lebensqualität für die jungen Ärztinnen und Ärzte.

Was früher nebenher geleistet worden war, bedeutete nun Arbeit in zwei Schichten von zwölf bis dreizehn Stunden. Die Wochenenden wurden weiterhin durch nur unvollständig kompensierte Dienste aufgefangen, weswegen die Stellenvermehrung moderat ausfiel. 
In der Kinderchirurgie wurde die Stellenvermehrung v.a. durch nicht operierende Pädiater und Allgemeinpraktiker getragen, so dass die Zahl der Eingriffe nicht durch eine grössere Zahl Ärzte geteilt werden musste und deswegen die Zeit bis zur Erfüllung des Operationskataloges nicht wesentlich verlängert wurde.

Negativ fiel aber auf, und dies gilt für alle Fachgebiete, nicht nur für die Kinderchirurgie, dass die Arbeit auf der Station zu leiden begann. Durch das Tag-/Nachtarzt-System und die teilweise Kompensation von Wochenenddiensten war eine kontinuierliche Stationsarbeit nicht mehr möglich. Die Stationsärzte wechselten häufig und konnten sich nicht mehr mit der Abteilung und ihren Patienten so identifizieren wie früher, für die Pflegenden waren sie nicht mehr die alleinigen Ansprechpersonen und auch die Patienten und ihre Eltern beklagten sich über die mangelnde Kontinuität. Beträchtliche Zeit wurde für Übergaben, Übernahmen und Einführungen aufgewendet und die Visite mit dem Zettel in der Hand, welcher vom Vorgänger geschrieben worden war, wurde ein gewohntes Bild. Die Oberärzte, früher viel weniger auf der Station präsent und die Arbeit der Stationsärzte lediglich überwachend, sprangen ein und übernahmen einen Teil der Stationsarbeit. Dadurch wurden der Oberarzt und die Oberärztin direkte Ansprechpartner für Pflegende und Eltern, was die Stellung des Arztes in Weiterbildung weiter schwächte. Da gleichzeitig die Anforderungen stiegen, Wartezeiten auf der Station und im Notfall nur schlecht toleriert wurden und rascher nach dem Oberarzt gerufen wurde, sank das Prestige des Arztes in Weiterbildung zusehends. «Nur der Assistenzarzt» wurde eine häufig gehörte Floskel. Deswegen brachten die Kandidatinnen und Kandidaten nicht mehr dieselbe Behandlungskompetenz an die Prüfung mit wie ihre Vorgänger.

\section{Präsenz- und Arbeitszeiten}

Die durchschnittliche Präsenzzeit im Spital, selbstverständlich inklusive Ruhezeiten, betrug 1980 rund 3400 Stunden pro Jahr, was etwa 80 Wochenstunden entspricht. Die Zeit der spezifischen Weiterbildung betrug damals rund vier Jahre. Seitdem ist die Präsenzzeit kontinuierlich auf etwa 2400-2500 Jahresstunden, 55-60 Wochenstunden Arbeit und Präsenz, gefallen. Die heutigen Fachärztinnen und Fachärzte für Kinderchirurgie erhalten den Facharzttitel nach durchschnittlich 9-10 Jahren, was einer spezifischen Weiterbildungszeit von 5 bis 6 Jahren entspricht.
Was wird ab 2005 geschehen? Dauert die spezifische Weiterbildung nochmals zwei Jahre länger? Ist eine totale Weiterbildungszeit von zwölf Jahren noch sinnvoll? Muss man 40 Jahre alt werden, um einen Facharzttitel für Kinderchirurgie zu erwerben? Und wie alt muss man werden, um kompetent und alleinverantwortlich tätig zu sein?

Oder kollabiert das System, wie ein Velofahrer schliesslich von seinem Vehikel fällt, wenn er zu langsam den Berg hinauffährt?

Die maximale wöchentliche Präsenzzeit im Spital, inklusive 1 Stunde Znüni-, Mittags- und Zvieripause pro Tag, beträgt 50 Stunden. Da die interne Weiterbildung weitere 2-3 Stunden pro Woche umfasst, unterscheidet sich die Arbeitszeit der Assistenzärztinnen und Assistenzärzte nicht mehr wesentlich von den in der Schweiz für alle Arbeitnehmer üblichen 42 Stunden. Auch hat ein Assistenzarzt, wie alle anderen Angestellten, Anrecht auf 4 Wochen Ferien und rund 15 Feiertage pro Jahr.

Dazu kommt das Recht auf externe Weiterbildung, welche weitere 50 Stunden pro Jahr beansprucht. Nicht berücksichtigt in dieser Aufstellung ist die Zeit, in welcher der Arzt in Weiterbildung unter direkter Aufsicht, sei es im Operationssaal oder auf der Abteilung, arbeitet. Auch hier handelt es sich um Weiterbildungszeit, könnte doch der mitarbeitende Vorgesetzte die Aufgabe schneller allein erfüllen.

Erschwerend kommt hinzu, dass die maximale Zykluslänge 6 Tage beträgt. Schichtlängen von mehr als neun Stunden sind nicht erlaubt, nach dieser Zeit muss eine mindestens einstündige ungestörte Pause eingelegt werden können. Dies bedeutet, dass ein Arbeitsrhythmus $5+2$, 5 Arbeitstage und ein Wochenenddienst, wie er auf der Station und im Operationssaal die Regel ist, nicht mehr eingehalten werden kann und die kurzen Mittagspausen, in welchen der Assistenzarzt bei Bedarf für die Weiterarbeit im Operationssaal zur Verfügung stand, der Vergangenheit angehören.

\section{Kann der Weiterbildungsauftrag noch erfüllt werden?}

Es stellt sich nun immer dringender die Frage, ob der Weiterbildungsauftrag unter den genannten Umständen noch erfüllt werden kann.

Nehmen wir als Beispiel die Chirurgie an Neugeborenen, ein Kerngebiet der Kinderchirurgie.

Die Zahl der Neugeborenen, welche operiert werden müssen, geht dank Prophylaxe (Folsäure), aber auch wegen der pränatalen Diagno- 
stik und damit einhergehend Schwangerschaftsunterbrechungen bei bekannten Fehlbildungen, zurück. Wir rechnen zurzeit in der Schweiz mit 300-400 Operationen an Neugeborenen pro Jahr. Diese Operationen werden ausnahmslos in kinderchirurgischen Kliniken von Kinderchirurgen durchgeführt. Die Operationen finden z.T. notfallmässig Tag und Nacht (Darmperforationen) statt und verteilen sich auf rund 10-15 Ärztinnen und Ärzte in Weiterbildung. Die Zahl 20-40 pro Kandidat oder 60-120 während einer Weiterbildungsperiode sollte eigentlich für eine kompetente Weiterbildung genügen. In Wirklichkeit ist der Arzt in Weiterbildung aber wegen der verkürzten Arbeitszeit nur noch etwa 3 von 5 Tagen im Spital, verpasst also notgedrungen $40 \%$ aller Operationen an Neugeborenen. Zudem muss der Kandidat viele Eingriffe assistieren, bis er das erste Mal unter Aufsicht ein Neugeborenes operieren darf. So wird rasch klar, dass auch unter besten Voraussetzungen die 31/4 Jahre der spezifischen Weiterbildung für die geforderte Fachkompetenz nicht reichen können.

Dasselbe gilt nicht nur für die Chirurgie an Neugeborenen, sondern ebensosehr für andere rekonstruktive oder traumatologische Eingriffe.

Dank der Tätigkeit des Schulpolizisten, aber auch dank der Trennung von Strasse und Spiel, der Geschwindigkeitsbeschränkungen, der Helme und anderer Faktoren ist zum Glück die Zahl der schweren Unfälle deutlich zurückgegangen. Deswegen muss ein Weiterbildungskandidat manchmal Wochen warten, bis er wieder Gelegenheit erhält, einen schwerverletzten Patienten im Schockraum zu behandeln.

Die Aufnahme eines Patienten auf der Notfallstation, die Anwesenheit bei der anschliessenden Operation und die Nachbetreuung auf der Abteilung, früher ein gängiges Vorgehen, wird durch rigide Dienstpläne vereitelt. Nicht zu Unrecht hat ein Transplantationschirurg einmal gesagt, dass nur derjenige kompetent einen Patienten nach einer Transplantation betreuen kann, welcher die ersten Tage ununterbrochen in der Nähe des Patienten verbracht hat und damit die Symptome sich anbahnender Katastrophen kennenlernt. Deswegen müssen immer mehr Behandlungsschemata produziert werden, da die natürliche im Operationssaal und auf der Abteilung erworbene Erfahrung fehlt.

Aber auch die tägliche Routine leidet, indem die Ärztinnen und Ärzte immer weniger dazu kommen, auch einfachste Eingriffe selbst durchzuführen. Blutentnahmen werden, wenn immer möglich, vermieden und wenn sie doch einmal notwendig werden, wird gleich der Anästhesist gerufen, da sich der Arzt nicht mehr getraut, für eine Blutentnahme vielleicht zweimal stechen zu müssen. Dadurch entstehen unproduktive Wartezeiten auf der Notfallstation wie auf der Abteilung. Rapportzeiten müssen neu eingeführt und bestehende verlängert werden, damit alle auf dem gleichen Wissensstand sind. Was dies bedeutet, hat uns der Pflegedienst in den letzten 20 Jahren vorgelebt, ein nicht erstrebenswertes Beispiel.

Das Jahr hat 8760 Stunden, deswegen benötigt man unter Berücksichtigung der Nachtarbeitszeit mit ihren Kompensationen mindestens 4 Stellen à 2250 Stunden, um Tag und Nacht einen Arzt oder eine Ärztin in der Notfallstation zu haben. In einer grossen Klinik mit 40 Assistenzärzten und Assistenzärztinnen ist das nicht so schlimm. Kinderchirurgische Kliniken aber sind nie so gross. In meiner Klinik mit 7 Weiterbildungsstellen bedeutet dies, dass zwei Drittel der gesamten assistenzärztlichen Kapazität und damit der Jahresarbeitszeit für die Notfallstation verbraucht werden müssen. Selbstverständlich kann dort ein Teil der Weiterbildung erfüllt werden. Das Nähen von Rissquetschwunden und die Reposition von Frakturen genügt aber nicht. Sämtliche komplexen Eingriffe und alle Wahloperationen beschränken sich auf das letzte Drittel der Jahresarbeitszeit. Gegenüber einem Kollegen, welcher vor 20 Jahren seine Facharztausbildung absolvieren konnte und die Arbeit in der Notfallstation und am Wochenende nebenher erledigte, bedeutet obige Rechnung, dass sich die spezifische Weiterbildungszeit mindestens verdoppeln muss! Und diese Rechnung geht nur dann auf, wenn heute wie vor 20 Jahren gleich viele Eingriffe auf einen Weiterbildungskandidaten kommen!

\section{Lösungsansätze}

Nun, das Gesetz ist geschrieben, wir haben uns nicht rechtzeitig gewehrt und müssen uns nun anpassen und Lösungsansätze finden.

Der grosse Brocken, die 4-5 Stellen der Notfallstation, muss verkleinert werden. Dies bedeutet, dass z.B. mehrere Kliniken zusammen eine Notfallstation betreiben. Im Kinderspital Luzern werden wir voraussichtlich die kinderchirurgische und die pädiatrische Notfallstation zusammenlegen und den kinderchirurgischen Assistenzarzt nachts in den in der Regel schwach frequentierten Stunden von der Notfallstation abziehen.

Konsequenzen sind längere Wartezeiten für die Patienten und deren Eltern, da sich weniger 
Ärzte um vorläufig gleich viele Patienten kümmern müssen, schlechtere Fachkompetenz, da Ärzte Auskunft geben müssen, welche das Fach nicht kennen und eine vermehrte Belastung der Kaderärzte, welche auch für kleinste Eingriffe ins Haus kommen müssen. Weiter wird versucht werden, den Weiterbildungskandidaten möglichst wenig auf der Notfallstation zu beschäftigen und diese Arbeit durch Ärztinnen und Ärzte mit anderen Berufszielen oder mit Spitalärzten $\mathrm{zu}$ besetzen.

Da die Tagesarbeitszeit beschränkt ist, müssen Zeit im Operationssaal und Zeit auf der Station gegeneinander abgewogen werden. Stationssekretärinnen können nur einen Teil der administrativen Arbeiten übernehmen. Um einen reibungslosen Betrieb zu gewährleisten, geht die Arbeit auf der Station der Tätigkeit im Operationssaal vor. Dies wird von Eltern und Patienten, aber auch vom Pflegedienst und von den einweisenden Ärzten verlangt.

Die Folge aus diesen Forderungen besteht schon heute darin, dass die operierenden Kaderärzte immer häufiger mit Unterassistentinnen und Unterassistenten in den Operationssaal gehen. Zwar entspricht dies nicht deren Ausbildungsniveau und ist vielleicht in bezug auf den Staatsexamensstoff nicht immer sinnvoll, gibt den Unterassistenten aber eine Aufgabe und hilft mit, den Klinikbetrieb aufrechtzuerhalten. Nachteilig wirkt sich dieses Vorgehen natürlich für die Assistenzärztinnen und Assistenzärzte aus, welche, obwohl in einer chirurgischen Klinik tätig, kaum mehr operative Eingriffe miterleben.

Um all die kleineren und grösseren Friktionen aufzufangen, braucht es Kaderärzte, welche weiter am Karren ziehen. Sie werden an erster Stelle die Leidtragenden sein, da sie versuchen werden, irgendwie die Arbeit so weiterzuführen, wie sie es gelernt haben und wie sie es lieben.

Trotzdem besteht kein Zweifel daran, dass die Qualität der Weiterbildung sowohl für Chirurgen wie für Grundversorger sinken wird, dass aber auch die Qualität der Klinikarbeit, also der Betreuung unserer Patienten, leidet.

\section{Vorschläge}

Um die Weiterbildung trotzdem zu gewährleisten, müssen einerseits rigide Vorschriften für die Ärztinnen und Ärzte in Weiterbildung und für die Kliniken erlassen werden und andererseits dieser spezifischen Weiterbildung die Weiterbildung der anderen Ärztinnen und Ärzte untergeordnet werden. Nur so können wir errei- chen, das auch in Zukunft unsere Patientinnen und Patienten kompetent versorgt werden.

1. Die Zahl der Ärzte in Weiterbildung zum Facharzt für Kinderchirurgie wird auf maximal einen pro A-Klinik, also 7 in der Schweiz, beschränkt.

2. Das Weiterbildungsprogramm der Klinik wird auf diesen Kandidaten ausgerichtet.

3. Es wird ein gesamtschweizerischer Pool eingerichtet, damit die Kandidaten durch möglichst viele Operationen mit einem möglichst grossen Spektrum geschleust werden können.

Bei 7 Ärztinnen und Ärzten in Weiterbildung und einer Weiterbildungszeit von $3 \frac{1}{2}$ Jahren könnten so jedes Jahr zwei Fachärzte «produziert» werden. Rechnen wir mit rund 50 Fachärzten in Spitälern und Praxen und einer durchschnittlichen Lebensarbeitszeit von 25 Jahren nach dem Erreichen der Facharztanerkennung, geht die Rechnung genau auf.

Wir müssen aber damit rechnen, dass in Zukunft auch für Kaderärzte die 50-StundenWoche gelten wird und dass von den jungen Ärztinnen und Ärzten neue und andere Arbeitsmodelle gewählt werden, welche nicht mit einer $100 \%$-Anstellung einhergehen. So wird es auch unter optimalsten Bedingungen nicht möglich sein, die notwendige Zahl an Kinderchirurgen weiterzubilden. Die Folge wird in kurzer Zeit ein gravierender Mangel an Kaderpersonal in den Spitälern und an Ärzten in der freien Praxis sein.

Da die bilateralen Verträge unterzeichnet sind und in Europa die Freizügigkeit in bezug auf den Arbeitsplatz herrscht, müssen wir uns fragen, ob ausländische Kolleginnen und Kollegen die Lücken füllen können?

Abgesehen davon, dass die einheimische Bevölkerung nicht unbedingt begeistert ist, wenn ausländische Ärzte ihre Kinder behandeln (und die Balkanstaaten, die Türkei und Sri Lanka sind nicht in der EU), stellt sich die Frage, ob diese die gestellte Aufgabe überhaupt übernehmen können. Während in vielen europäischen Ländern Kindertraumatologie und Kinderurologie nicht von Kinderchirurgen durchgeführt werden, sind dies in der Schweiz wichtige Gebiete des Faches, welche nicht von Chirurgen für Erwachsene übernommen werden können. Zudem gibt es auch im benachbarten Ausland zu wenige Kinderchirurgen, so dass wir rasch mit einem gravierenden Mangel an Fachärztinnen und Fachärzten konfrontiert werden. 


\section{Alternativen}

Welche Alternativen haben wir?

\section{Die Ansprüche an den Anwärter für den Facharzttitel Kinderchirurgie werden reduziert}

Der Titel bedeutet nicht mehr breites Wissen und chirurgisches Können auf hohem Niveau, sondern Basiswissen für den Alltag. Grosser Wert müsste auf die Traumatologie gelegt werden, da etwa die Hälfte aller Patientenkontakte verunfallten Kindern gilt. Im Anschluss an den Facharzttitel müssten neue Schwerpunkte geschaffen werden. Ich denke hier z.B. in Analogie zu den Urologen an einen Schwerpunkt «operative Kinderchirurgie», welcher weitere zwei Weiterbildungsjahre beanspruchen würde. Eine andere Lösung würde darin bestehen, Subspezialitäten wie Unfallchirurgie, Urologie oder Neugeborenenchirurgie zu Schwerpunkten zu erklären.

\section{Es wird auf die nicht fachspezifische Weiterbildung verzichtet}

Damit hätten wir 23/4 Jahre länger Zeit, die Kandidatinnen und Kandidaten zu Fachärzten weiterzubilden. Deutschland hat dies vorgemacht und in den vergangenen Jahren versucht, ihre Titelanwärter sechs Jahre lang nur in Kinderchirurgie weiterzubilden. Schon bald wird diese Entwicklung wieder rückgängig gemacht werden, da das Basiswissen in Chirurgie viel leichter und schneller in der Erwachsenenchirurgie als in der Kinderchirurgie gelernt wird. Chirurgische Zugänge, Wundverschlüsse, Legen von Blasenkathetern oder von Thoraxdrainagen, endoskopische Verfahren sind in der Erwachsenenchirurgie viel häufiger als in der Kinderchirurgie und müssen deswegen dort gelernt werden. Der Verzicht auf Pädiatrie und Anästhesie könnte weitere neun Monate bringen, würde aber mit dem Verlust an Behandlungskompetenz in bezug auf pädiatrische Erkrankungen bezahlt.

\section{Die Weiterbildungszeit gilt nicht mehr als Arbeitszeit}

Die jungen Ärztinnen und Ärzte werden für eine 42-Stunden-Woche angestellt, in welcher sie, wie jeder andere junge Akademiker auch, für ihren Betrieb arbeiten. Jede assistierte Operation, jede Besprechung eines Patienten mit dem Oberarzt und alle externen und internen Lehrveranstaltungen gelten nicht als Arbeit, sondern als Weiterbildung. Es ist zu prüfen, ob ein solches System den gesetzlichen Vorgaben standhält. Es würde aber die Stellung der Assistenzärztinnen und Assistenzärzte stärken, welche dann wieder als Ärzte angesehen würden und nicht als Lehrlinge.

Ich sehe es als meine Aufgabe an, Ihnen als Vertreter unseres Gesundheitssystems, aber auch als Eltern von möglichen Patientinnen und Patienten zu zeigen, dass ein labiles Gleichgewicht mutwillig zerstört werden wird. Unsere Liebe für das Fach und unsere Hingabe an eine äusserst befriedigende Arbeit sind nicht verstanden worden. Dass ausgerechnet freisinnige Politiker an vorderster Front die Eigenverantwortung, auch für die Weiterbildung, nicht mehr wahrhaben wollen, ist bitter.

Ich kann Ihnen kein Rezept ausstellen, wie nun die Vorgaben der WBO unter veränderten Umständen eingehalten werden können. Ich plädiere dafür, dass wir uns endlich eingestehen, wie lange die Weiterbildung in Wirklichkeit dauert, dass wir diese Zeit akzeptieren und offiziell in die WBO aufnehmen. Weiter schlage ich vor, dass wir entweder einen «kleinen» Facharzttitel nach sechs Jahren verleihen und einen operativen Schwerpunkt schaffen, welcher nach weiteren 2 Jahren vergeben wird oder mehrere Schwerpunkte benennen, welche in zwei zusätzlichen Jahren erreicht werden können.

Weltfremde Gesetze, welche die Besonderheiten unseres Faches nicht berücksichtigen, dispensieren uns nicht davon, unsere kleinen Patienten kompetent zu behandeln. 